\title{
Automated robot based systems for crystallography on beamlines and in laboratories, and other developments performed on FIP-BM30A at the ESRF
}

\author{
J-L Ferrer, X Vernede, Y Sallaz-Damaz, C Berzin, P Jacquet, M Budayova-Spano, D Cobessi, F Borel
}

IBS Univ. Grenoble Alpes, CEA, CNRS, F-38000 Grenoble, France.

Beamline FIP-BM30A is a highly automated macromolecular crystallography (MX) beamline located at the European Synchrotron Radiation Facility (ESRF). On such a beamline, a significant number of users choose to manage their experiment remotely or in an automated/unattended mode. So, automation of protein crystallography experiments has always been an important goal for the FIP-BM30A beamline scientists since it started operation in 1999.

The control software now benefits from the so-called "WIFIP" 1 , a recently developed web-based user interface for MX beamlines. WIFIP provides a simplified, highly reactive graphical user interface, and is designed to be adapted very quickly to be able to integrate new features as the need arises.

On the instrument side, the development of 6-axis robotic arm based systems for protein crystallography continues to expand rapidly. From our seminal work to the present developments, robot based systems significantly changed the range of crystallography experiment strategies. Indeed, the first stage occurred in 2000 on FIP-BM30A when we implemented and began using the first 6-axis robot based sample changer for $\mathrm{MX}^{2}$. Today, FIP-BM30A has a full robotized platform up and running, on which the current developments are made. One of these, named G-Rob ${ }^{3}$, serves as a fully integrated, multi-purpose diffractometer for beamlines and laboratories, with goniometer capabilities. G-Rob integrates many functions: multi-standard (SPINE, Unipuck, miniSpine) sample changer (in operation on FIP since 2004); goniometer for frozen samples or capillaries ${ }^{3}$; crystallization trays handling for in situ screening and data collection on crystallization plates and microchips ${ }^{4}$ (in operation on FIP since 2006); powder diffraction; beam monitoring; crystal harvesting (under development); etc. Thanks to its tool changer, G-Rob's robotic arm can automatically switch from one function to the other. G-Rob can also easily be upgraded with the addition of new functions, such as crystal harvesting, presently under development. ${ }^{6,7}$ Several G-Rob systems, both at synchrotrons or as laboratory in-house systems, are currently available for use by

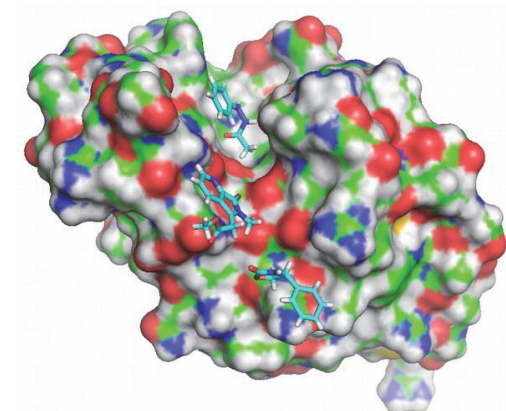
the crystallography community (N.B. G-Rob is commercialized by NatX-ray under an exclusive CEA-CNRS license).

Latest result using G-Rob: Automated in situ diffraction ligand screening on beamline FIP-BM30A. The target protein crystals are reproduced all over a crystallization plate previously pre-coated ${ }^{5}$ with fragments from a library optimized for crystallography. Crystallography data are systematically collected in situ for each fragment, using a fully automated pipeline ${ }^{8}$. First "real scale" tests revealed several hits (see figure).

At present, the FIP-BM30A team also focuses on rational crystallization, with a novel instrument that makes possible the implementation of kinetic trajectories in the multi-dimensional phase diagram. This instrument, named Opticrys $^{9}$, is currently available on a collaboration basis to ESRF MX beamline users and ILL users.

\footnotetext{
${ }^{1}$ Sallaz-Damaz, Y. and Ferrer, J.-L. (2017). J. Synchrotron Rad. 24(5), 1105-1111.

${ }^{2}$ Ohana et al., J. Applied. Cryst. 37(1) (2004), 72-77.

3 Jacquamet et al., Acta Cryst. D60 (2004), 888-894.

${ }^{4}$ Jacquamet et al., Structure 12 (2004), 1219-1225.

${ }^{5}$ Gelin et al., Acta Cryst. D 71 (2015), 1777-1787.

${ }^{6}$ Heidari Khajepour et al., Acta D69 (2013), 381-387.

${ }^{7}$ http://www.msrl.ethz.ch/research/current-research/protein-crystal-harvesting.html

${ }^{8}$ Schiebel et al., Structure 24 (2016), Issue 8, 1398-1409.

${ }^{9}$ Junius et al., Applied Crystallography 49 (2016), 806-813.
} 